\title{
Analysis and Comparison the Dynamical Properties for Two Hyperchaotic Systems
}

\author{
Saad Fawzi Jasim AL-Azzawi Ahmed Intisar Ghitheeth \\ Department of Mathematics \\ College of Computer Sciences and Mathematics \\ Mosul University
}

Received

12/06/2012
Accepted

$16 / 10 / 2012$

\begin{abstract}
الملخص
في هذا البحث تم اقترح نظام جديد رباعي الإبعاد مستمر مستقل ذاتيا كثير

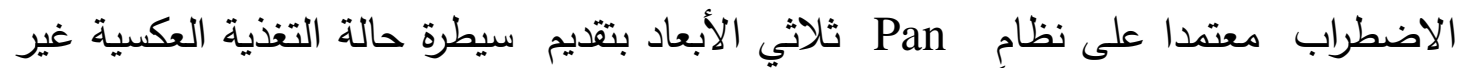

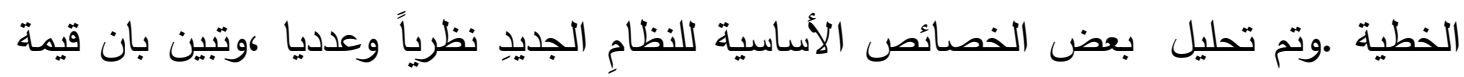

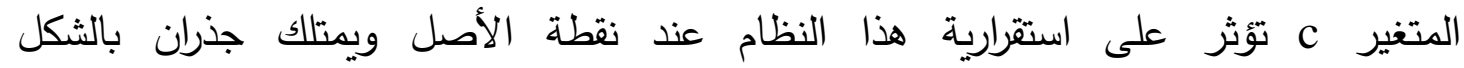
خصانص خصائص نظام أخر رباعي الإبعاد كثير الاضطراب وبين الاختلاف بينهما وأخيرا تم إعطاء • مثال توضيحي
\end{abstract}

\begin{abstract}
In this paper, a new four-dimensions hyperchaotic system is proposes based on a three-dimensional Pan system by introducing a nonlinear state feedback controller, and we investigated some basic properties for this system numerically and analytically, we found the parameter $\mathrm{c}$ is effective on stability of new system at the point $O(0,0,0,0)$ and has two roots form $\lambda_{1}=-b, \lambda_{2}=-d \quad$ while has root $\lambda_{1}=0$ at the points $p_{1,2}$, and we compare the properties of a new system with properties of another 4D hyperchaotic system and show the difference between them, Finally, an illustrative example is given.
\end{abstract}

Keywords: Hyperchaos, Lyapunov exponent, Pan system , Stability,

\section{1- Introduction:}

Hyperchaos generation and control have been extensively studied in recent years, due to its theoretical and practical applications in 
the fields of communications, laser, neural work, nonlinear circuit, mathematics, and so on[11].

In mathematics and physics, chaos theory describes the behavior of certain nonlinear dynamical systems that under specific conditions exhibit dynamics that are sensitive to initial conditions(popularly referred to as the butterfly effect)[3].

Hyperchaotic systems have received much attention in recent years, particularly the hyperchaotic Rössler system and its variation. that is, the noted four-dimensional(4D) hyperchaotic system[3,7,11,13], hyperchaotic systems are more suitable for some special engineering applications such as chaos based encryption and secure communication[3]. hyperchaotic system is usually defined as a chaotic system with at least two positive exponents[2,3,4,7,8,10]and is expanded in two or more directions, So the hyperchaotic system is more complex than chaotic system[7,8,10,11].

This paper proposes a new four- dimensional continuous autonomous hyperchaotic system based on the 3D Pan system by introducing a nonlinear state feedback controller. Dynamical behaviors of the new system are analyzed, both theoretically and numerically, including equilibrium points, Lyapunov exponents spectrum stability and bifurcation .

\section{2- Historically Description for System:}

In 1963, Lorenz discovered the first chaotic system when he studied atmospheric convection[11], many interesting chaotic and hyperchaotic systems have been proposed, such as Lü system, Liu system and so on [14 ] .in 2008 ,Yang and Chen proposed a 3D chaotic system with six terms including only two quadratic terms in a form very similar to the Lorenz, Chen and Lü systems based on the Lorenz system, where $a>0, b>0$ and $\mathrm{c}$ are constants [12].

In 2010 Pan et al. proposed another 3D chaotic system which in fact was similar to the system of Yang and Chen but the parameters $a, b$ and $\mathrm{c}$ are real constants, this system is described by

$$
\left.\begin{array}{l}
\dot{x}=a(y-x) \\
\dot{y}=c x-x z \\
\dot{z}=x y-b z
\end{array}\right\}
$$

Recently, this system is called Pan system or L $\ddot{u}$-like system[5,6] . [4] generated 4D hyperchaotic system (2009) based on system (1) and generated conditions and hyperchaotic system is described by 
(2)

$$
\left.\begin{array}{l}
\dot{x}=a(y-x) \\
\dot{y}=c x-x z+u \\
\dot{z}=x y-b z \\
\dot{u}=-d x
\end{array}\right\}
$$

where $a, b, c$ and $\mathrm{d}$ are constant parameters, when $a=35, b=3, c=35$ and $d=8$, system (2) has a hyperchaotic attractor as shown in fig. 1 .

In 2011, Pan et al generated another 4D hyperchaotic system which has the following form[6]:

(3)

$$
\begin{aligned}
& \dot{x}=a(y-x)+u \\
& \dot{y}=c x-x z \\
& \dot{z}=x y-b z \\
& \dot{u}=-x z+d u
\end{aligned}
$$

When parameters $a=10, b=8 / 3, c=28$ and $d=1.3$, system (3) has a hyperchaotic attractor as shown in fig. 2

a

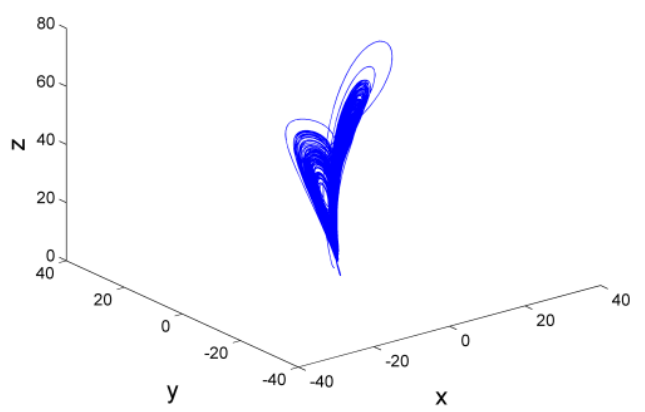

c

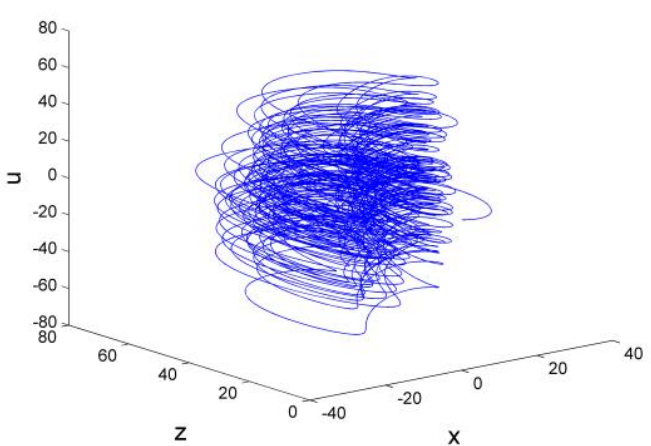

b

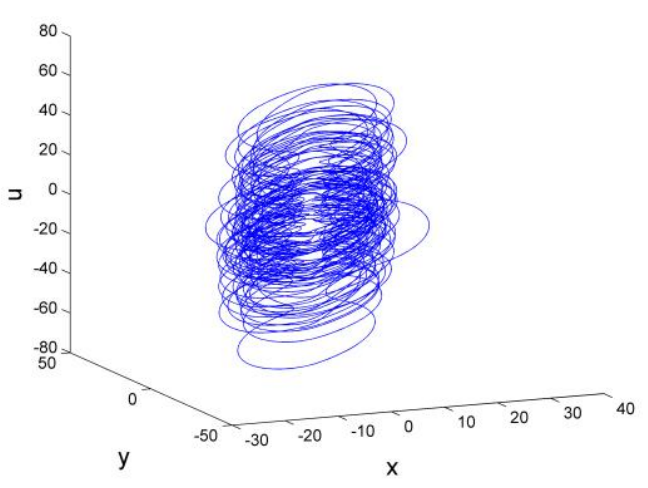

d

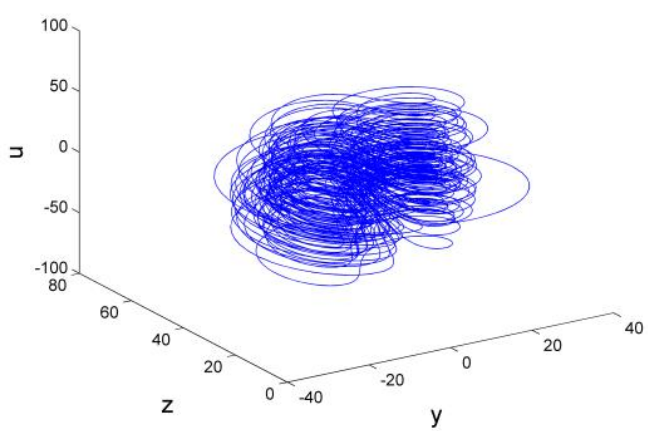

Fig. 1 Attractors of hyperchaotic system (2) in (a)the $x-y-z$ space;(b) the $x-y-u$ space, (c) the $x-z-u$ space, and(d) the $y-z-u$ space. 
a

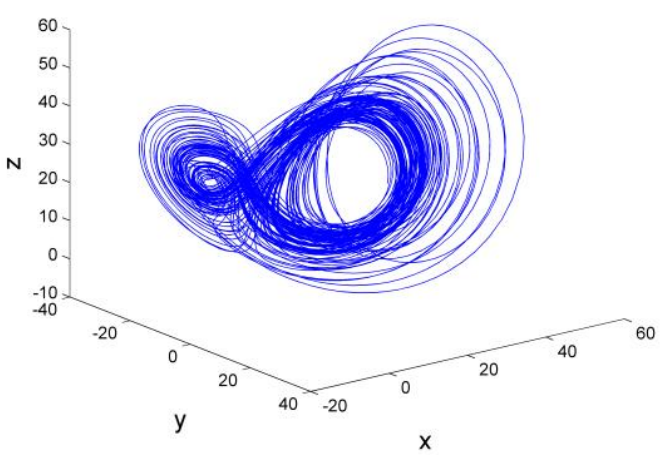

C

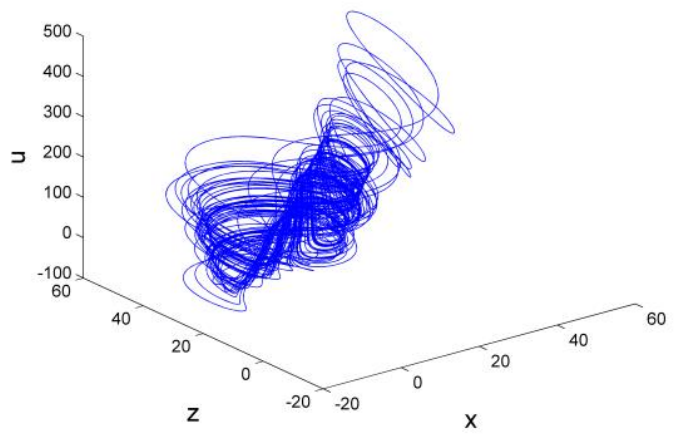

$\mathrm{b}$

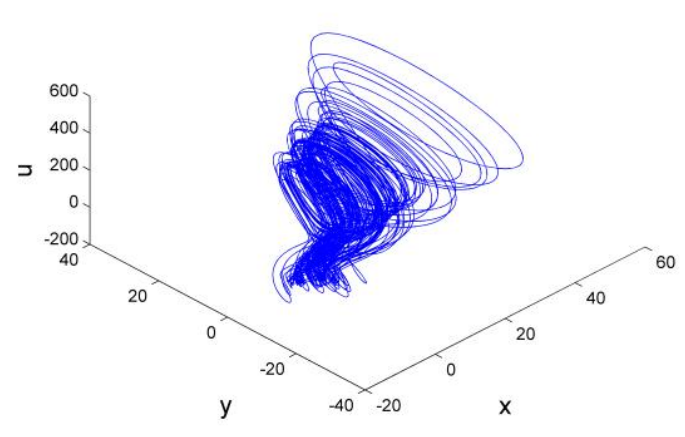

d

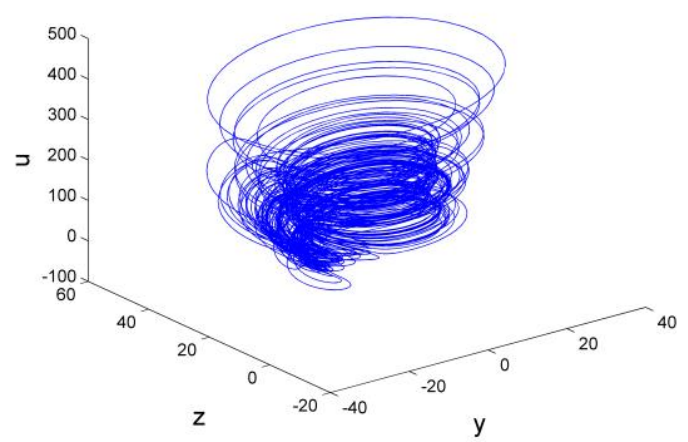

Fig. 2 Attractors of hyperchaotic system (3) in (a)the $x-y-z$ space;(b) the $x-y-u$ space, (c) the $x-z-u$ space, and(d) the $y-z-u$ space.

\section{3- Helping Results:}

Remark 1 (Lyapunov Exponents)[4,6] :

Lyapunov exponents by Wolf Algorithm are:

(i) in system (1) when $a=10, b=2$ and $c=16$ then $L E_{1}=0.8311, L E_{2}=0.0000$ and $L E_{3}=-12.5113$ and The Lyapunov dimension 2.0664[6].

(ii) in system (2) when $a=35, b=3, c=35$ and $d=8$ then $L E_{1}=0.2788, L E_{2}=0.1470, L E_{3}=0$ and $L E_{4}=-38.429$ [4] .

(iii) in system (3) when $a=10, b=8 / 3, c=28$ and $d=1.3$ then $L E_{1}=0.7340, \quad L E_{2}=0.2492, L E_{3}=0.0000$, and $L E_{4}=-11.3437$ [6].

The Lyapunov exponents spectrum of the systems $(1,2,3)$ are shown in Fig.3, Fig.4 and Fig.5 respectively 


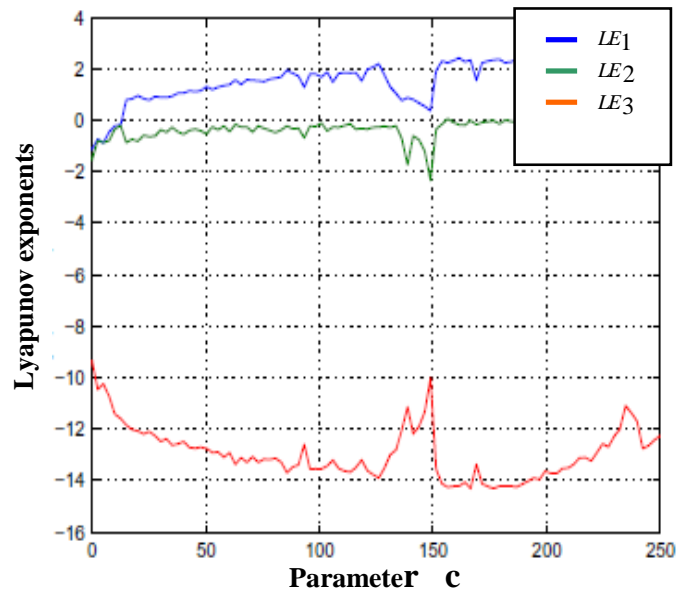

Fig. 3 Lyapunov exponents spectrum of system (1) versus parameter c

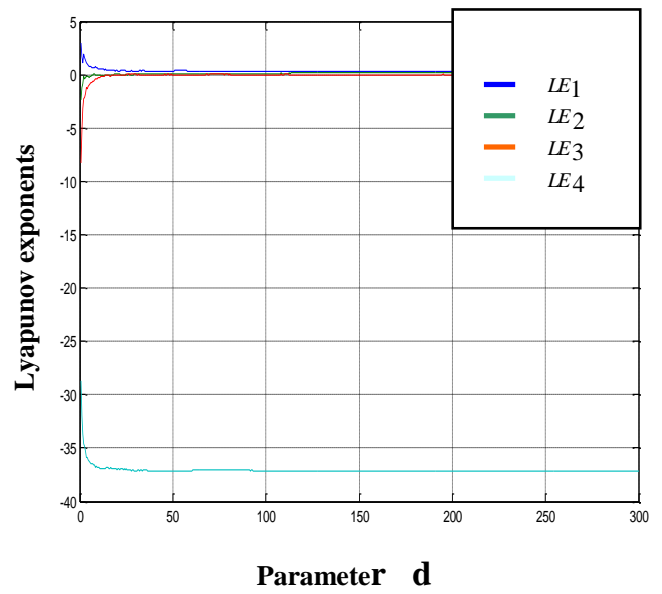

Fig. 4 Lyapunov exponents spectrum of system (2) versus parameter d

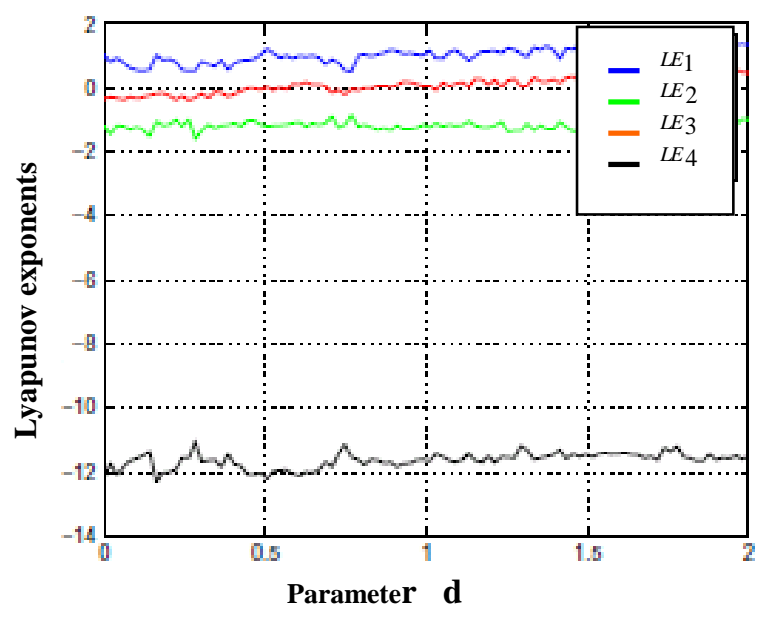

Fig. 5 Lyapunov exponents spectrum of system (3) versus parameter $d$

\section{Remark $2[4,14]$ :}

System (2) has unique equilibrium point $\mathrm{O}(0,0,0,0)$ and

(i) unstable always,

(ii) characteristic equation is of the form :

$$
f(\lambda)=\lambda^{4}+(a+b) \lambda^{3}+a(b-c) \lambda^{2}+a(d-b c) \lambda+a b d=0
$$

\section{Remark 3(Generating Hyperchaos) [2,6,7,9,11]:}

To generate hyperchaos from the dissipatively autonomously polynomial systems by using a state feedback controller, the state equation must satisfy the following two basic conditions:

(i) The minimal dimension of the phase space of an autonomous system is at least four. 
(ii) The number of terms in the coupled equations giving rise to instability is at least two, of which at least one has a nonlinear function .

\section{Remark 4 [1]: Critical Cases}

Critical cases in the theory of stability for differential equation means that cases when the real part of all roots of the characteristic equation are non-positive with the real part of, at least, one root being zero.

\section{Remark 5 (Routh- Hurwitz Test) [1]:}

All the roots of the indicated polynomial have negative real parts precisely when the given conditions are met.

- $\lambda^{2}+A \lambda+B: A>0, B>0$

- $\lambda^{3}+A \lambda^{2}+B \lambda+C: \quad A>0, C>0, \quad A B-C>0$.

- $\lambda^{4}+A \lambda^{3}+B \lambda^{2}+C \lambda+D: \quad A>0, \quad A B-C>0, \quad(A B-C) C-A^{2} D>0 \quad, D>0$.

In the context of ordinary differential equations ODEs the word "Bifurcation" has come to mean any marked change in the structure of the orbits of a system (usually nonlinear) as a parameter passes through a critical value[1].

\section{Remark 6(Hopf Bifurcation) [11]:}

Any system has a Hopf bifurcation if the following condition is satisfied:

1- The Jacobian matrix has two purely imaginary roots and no other roots with zero real parts.

$$
\text { 2- }\left.\frac{d}{d \mu}(\operatorname{Re}(\lambda(\mu)))\right|_{\mu=\mu 0} \neq 0
$$

\section{4- Main Results:}

Based on system(1) and remark 3,we can construct a new four dimensions hyperchaotic system by introducing a state feedback controller, as follows:

Add a nonlinear controller $u$ to the second equation of system (1), let $\dot{u}=z-d u$, then we obtain a new hyperchaotic system

$$
\left.\begin{array}{l}
\dot{x}=a(y-x) \\
\dot{y}=c x-x z+u \\
\dot{z}=x y-b z \\
\dot{u}=z-d u
\end{array}\right\}
$$


where $(x, y, z, u) \in R^{4}$, and $a, b, c, d \in R$ are constant parameters. For simplification, system(8) is called a new hyperchaotic system in this paper.

In the following we briefly describe some dynamical behaviors of a new hyperchaotic system (8).

\section{1 - Dissipative and Existence of Attracter:}

The system can be a dissipative system, because the divergenence of the vector field, also called the trace of the Jacobian matrix is negative if and only if the sum of the parameters $a, b$ and $d$ is positive, that is $a+\mathrm{b}+\mathrm{d}>0$

$$
\begin{aligned}
& \operatorname{div} \vec{V}=\frac{\partial \dot{x}}{\partial x}+\frac{\partial \dot{y}}{\partial y}+\frac{\partial \dot{z}}{\partial z}+\frac{\partial \dot{u}}{\partial u}=\operatorname{Tr}(J)=-(a+b+d), \\
& V(t)=V(0) e^{-(a+b+d) t}
\end{aligned}
$$

So, the system will always be dissipative if and only if when $a+\mathrm{b}+\mathrm{d}>0$ with an exponential rate: $\frac{d \vec{V}}{d t}=e^{-(a+b+d)}$.

\section{2- Equilibrium Points :}

In order to obtain the equilibrium points of $\operatorname{system}(8)$, let $\dot{x}=\dot{y}=\dot{z}=\dot{u}=0$, and we can obtain the following expressions(10):

$$
\left.\begin{array}{l}
a(y-x)=0 \\
c x-x z+u=0 \\
x y-b z=0 \\
z-d u=0
\end{array}\right\}
$$

From the above equations, we obtain three equilibrium points $O(0,0,0,0)$

$$
\begin{aligned}
& p_{1}\left(\frac{1+\sqrt{1+4 b c d^{2}}}{2 d}, \frac{1+\sqrt{1+4 b c d^{2}}}{2 d}, c+\frac{1+\sqrt{1+4 b c d^{2}}}{2 d^{2} b}, \frac{c}{d}+\frac{1+\sqrt{1+4 b c d^{2}}}{2 d^{3} b}\right), \\
& p_{2}\left(\frac{1-\sqrt{1+4 b c d^{2}}}{2 d}, \frac{1-\sqrt{1+4 b c d^{2}}}{2 d}, c+\frac{1-\sqrt{1+4 b c d^{2}}}{2 d^{2} b}, \frac{c}{d}+\frac{1-\sqrt{1+4 b c d^{2}}}{2 d^{3} b}\right) .
\end{aligned}
$$

\section{3- Lyapunov Exponents and Lyapunov Dimension :}

We calculate the Lyapunov exponents for a new hyperchaotic system with the Wolf Algorithm by using MATLAB software, the numerical simulation was carried out with $a=10, \quad b=8 / 3, \quad c=28, \quad d=1.3$ 
for initial value $(1,1,1,1)$ and the four Lyapunov exponents of the new hyperchaotic system(8)are $L E_{1}=0.17702, \quad L E_{2}=-0.027435, L E_{3}=-1.2336$, and $L E_{4}=-12.8815$. the Lyapunov exponents spectrum and attracters of system (8) in two and three dimensions are shown in Fig.6, Fig.7, and Fig.8 respectively.

So, we can obtain the Lyapunov dimension of the new hyperchaotic system(8),it is described as follows:

$$
\begin{aligned}
D_{L E}= & j+\frac{1}{\left|L E_{j+1}\right|} \sum_{i=1}^{j} L E_{i}=3+\frac{L E_{1}+L E_{2}+L E_{3}}{\left|L E_{4}\right|}= \\
& 3+\frac{0.17702-0.027435-1.2336}{|-12.8815|}=2.915847145
\end{aligned}
$$

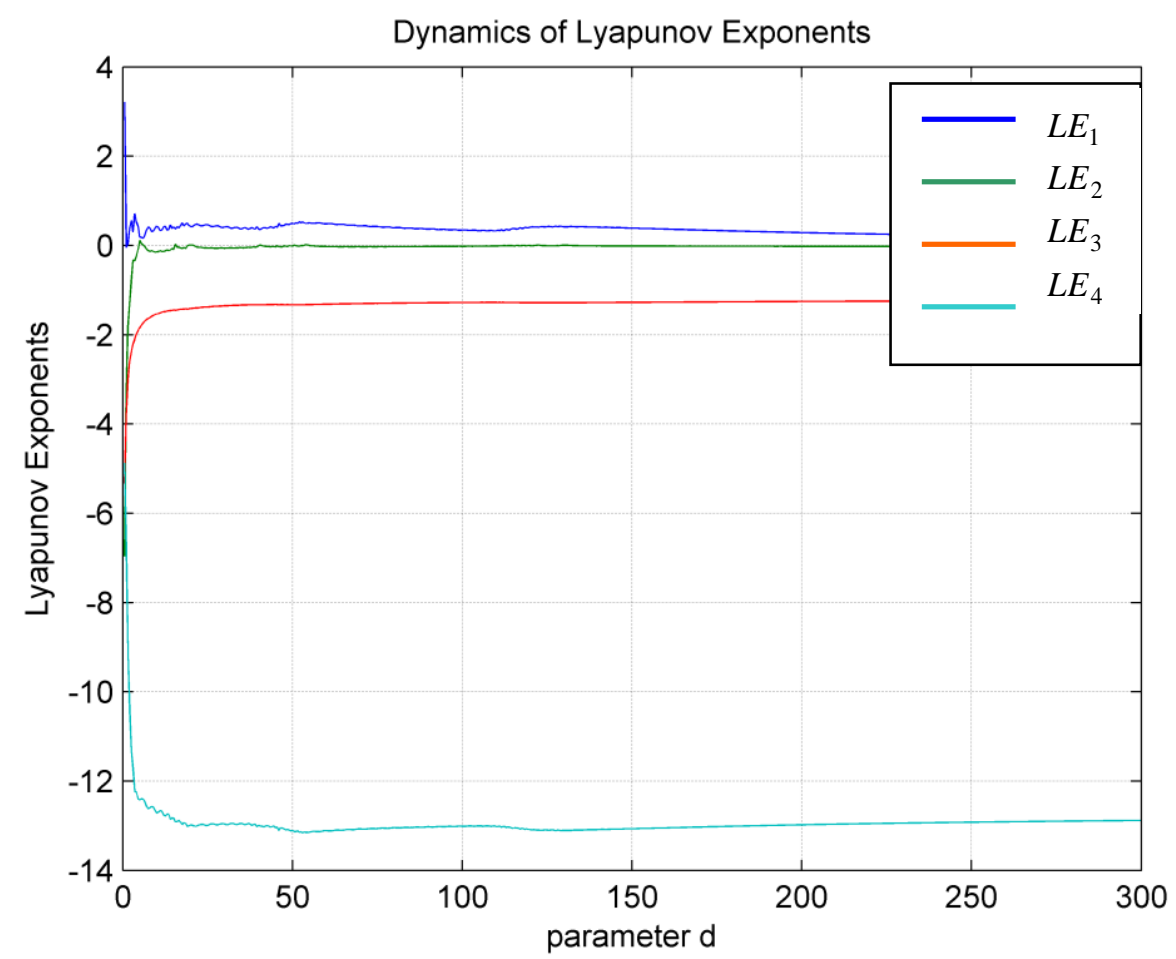

Fig. 6. Lyapunov exponents spectrum of new hyperchaotic system versus parameter $\mathrm{d}$ 

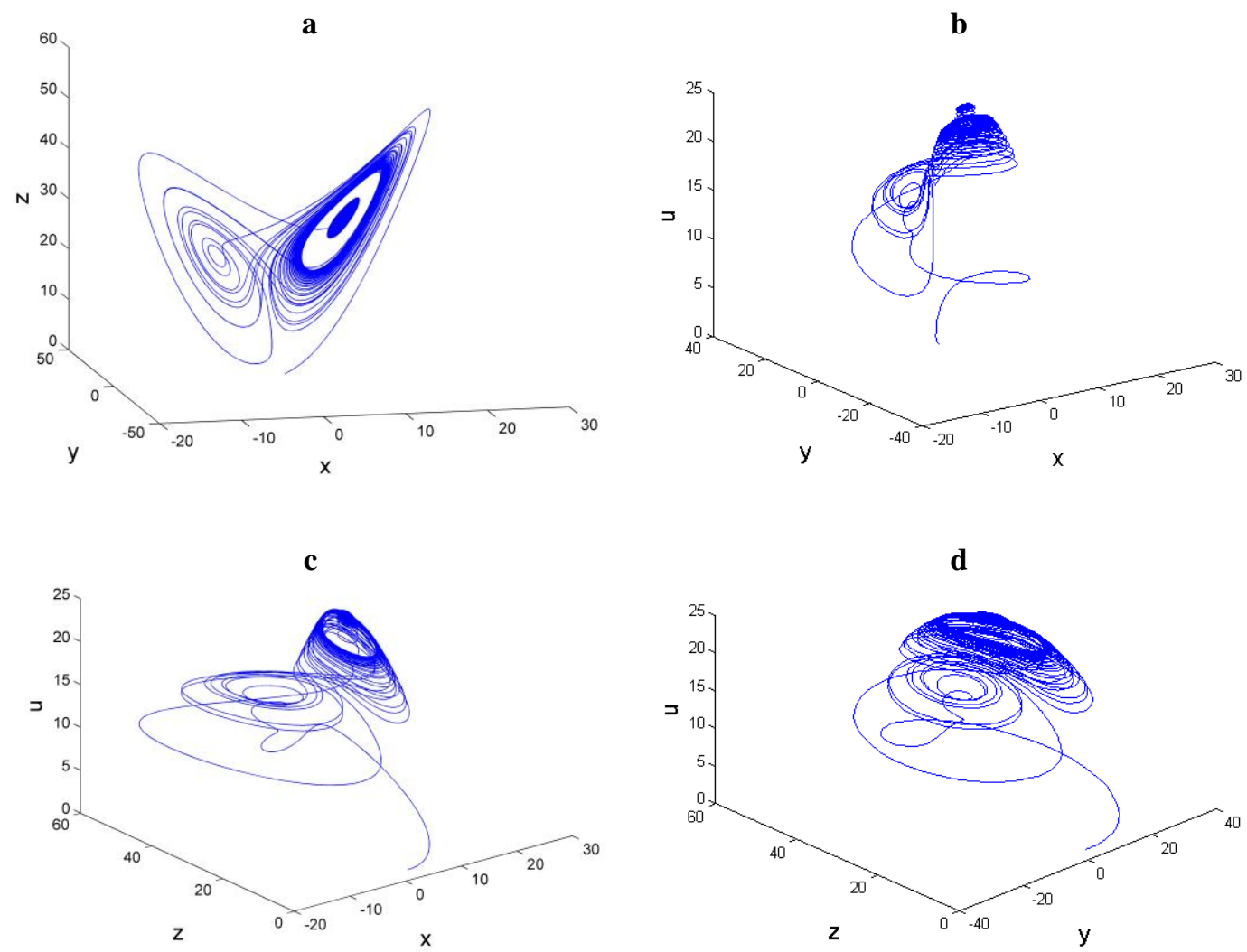

Fig. 7 Attractors of new hyperchaotic system in (a)the $x-y-z$ space;(b) the $x-y-u$ space, (c) the $x-z-u$ space, and(d) the $y-z-u$ space.
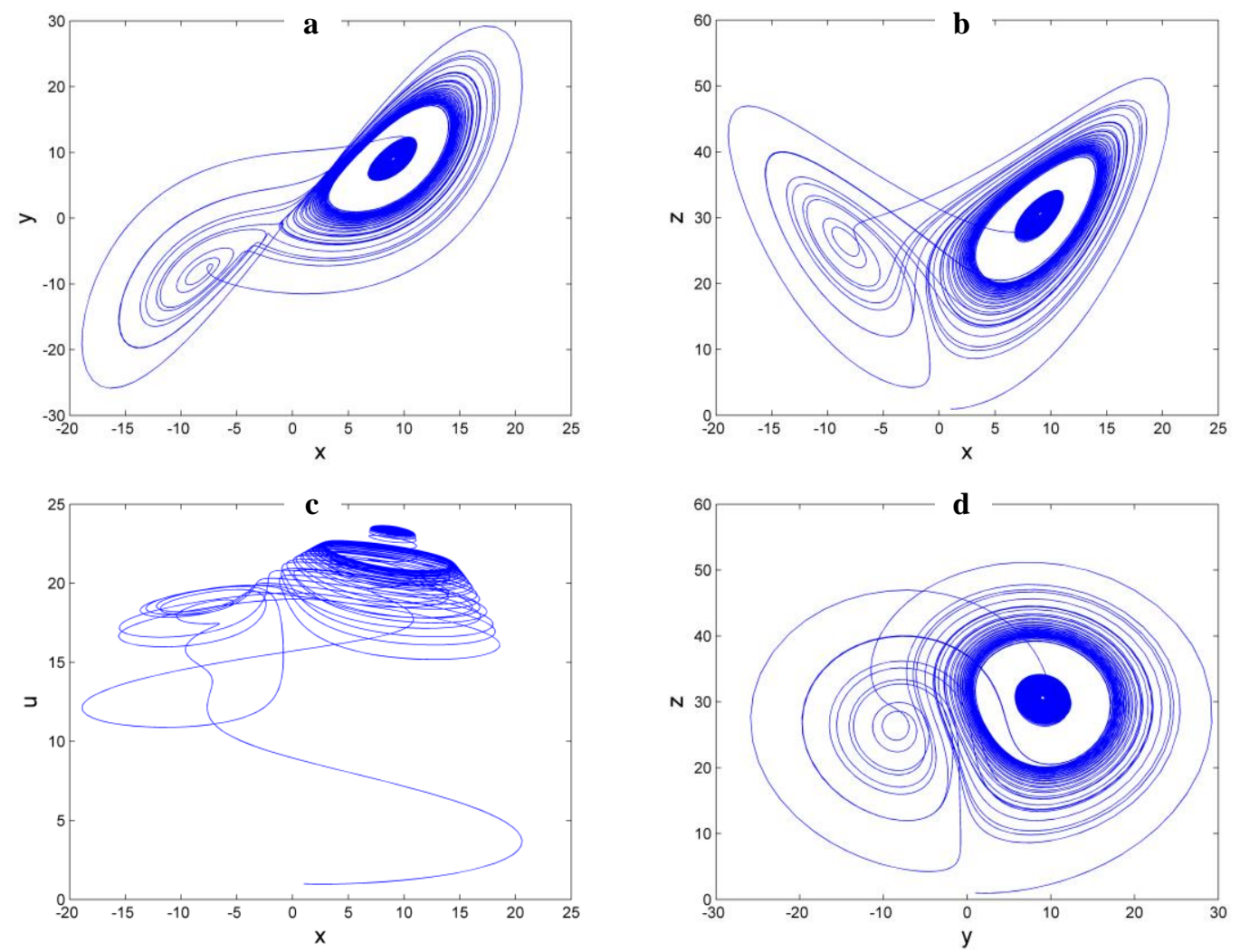

Fig. 8 Attractors of new hyperchaotic system in (a) $x-y$; (b) $x-z$; (c) $x-u$; (d) $y-z$.

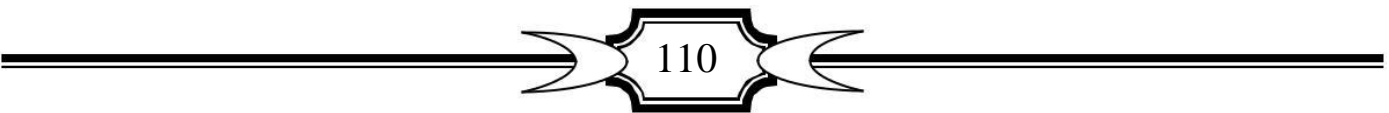


Theorem1: The solution of system (8) at the equilibrium point $O(0,0,0,0)$ when $a, b, d>0$ has the following cases:
1) Asymptotically stable
2) Unstable
if $\quad c<0$,
if $c>0$,

Proof: At the equilibrium point $O(0,0,0,0)$, system (8) is linearized, the Jacobian matrix defined as :

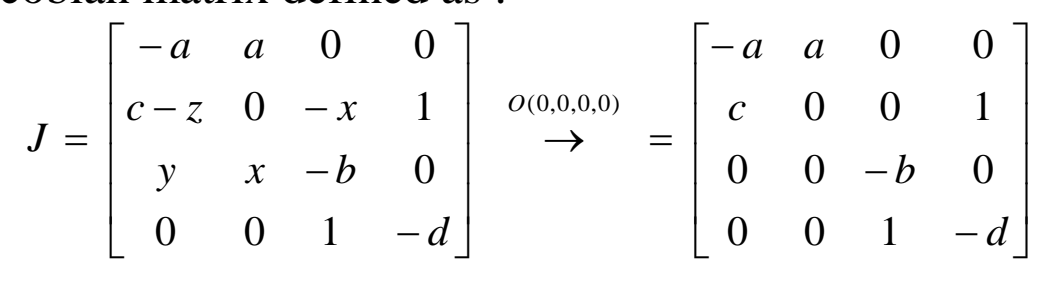

and its characteristic equation is :

$$
f(\lambda)=\lambda^{4}+(a+b+d) \lambda^{3}+(a b+a d+b d) \lambda^{2}-a c(b+d) \lambda-a b c d=0
$$

Solving equation (12) gives

$$
\lambda_{1}=-b
$$

and the following equation:

$$
f(\lambda)=\lambda^{3}+(a+d) \lambda^{2}+a(d-c) \lambda-a c d=0
$$

from equation (13) we get three eigenvalues corresponding to the equilibrium point $O(0,0,0,0)$ are:

$$
\lambda_{2}=-d, \lambda_{3,4}=\frac{-a \mp \sqrt{a^{2}+4 a c}}{2}
$$

So, it's clear that if $-a>\sqrt{a^{2}+4 a c}$, then $\lambda_{3}<0, \lambda_{4}<0$, But this is a possible under the condition $c<0$ since when $-a>\sqrt{a^{2}+4 a c}$ $\Rightarrow a^{2}>a^{2}+4 a c \Rightarrow 4 a c<0$ and $a>0$ given hence $c<0$, while $-a<\sqrt{a^{2}+4 a c}$ then $\lambda_{3}<0, \lambda_{4}>0$ is possible under the condition $c>0$.consequently the equilibrium point $O(0,0,0,0)$ is depended on parameter $\mathrm{c}$ to detriment the stability .

Theorem2 If $c=\frac{d(a+d)}{a}=c_{0},\left(c_{0}\right.$ is critical value $)$, then $\operatorname{system}(8)$ not Hopf bifurcation at the point $O(0,0,0,0)$.

Proof: If $c=\frac{d(a+d)}{a}=c_{0}$ the equation (13) is transformed into

$$
(\lambda+(a+d))\left(\lambda^{2}-d^{2}\right)=0
$$


with solutions $\lambda_{1}=-(a+d), \lambda_{2,3}= \pm d$, but this contradiction with the first condition of Hopf Bifurcation( remark6), Consequently, the system (8) not Hopf bifurcation.

Theorem3: The solution of system (8) at the equilibrium point $p_{1}$ is critical case .

Proof: Now to find Jacobian matrix at $p_{1}$ we need the following transformation

Under the linear transformation $(x, y, z, u) \rightarrow(X, Y, Z, U)$ :

$$
\left.\begin{array}{l}
x=X+x_{0} \\
y=Y+y_{0} \\
z=Z+z_{0} \\
u=U+u_{0}
\end{array}\right\}
$$

the system (8) becomes

$$
\left.\begin{array}{l}
\dot{X}=a(Y-X) \\
\dot{Y}=-\frac{1+\sqrt{1+4 b c d^{2}}}{2 d^{2} b} X-\frac{1+\sqrt{1+4 b c d^{2}}}{2 d} Z+U \\
\dot{Z}=-b Z \\
\dot{U}=\quad Z
\end{array}\right\}
$$

The equilibrium point $p_{1}$ of the system (8) is switched to the new equilibrium point $O^{\prime}(0,0,0,0)$ of the system (15) under the linear transformation,

The Jacobian matrix of the system $(15)$ at $O^{\prime}(0,0,0,0)$ is:

$$
J\left(O^{\prime}\right)=\left[\begin{array}{cccc}
-a & a & 0 & 0 \\
-\frac{1+\sqrt{1+4 b c d^{2}}}{2 d^{2} b} & 0 & -\frac{1+\sqrt{1+4 b c d^{2}}}{2 d} & 1 \\
0 & 0 & -b & 0 \\
0 & 0 & 1 & 0
\end{array}\right]
$$

and the characteristic equation is :

$$
\lambda^{4}+A \lambda^{3}+B \lambda^{2}+C \lambda=0
$$

where 


$$
\left.\begin{array}{l}
A=a+b \\
B=a b+\frac{1+\sqrt{1+4 b c d^{2}}}{2 d^{2} b} \\
C=a b\left(\frac{1+\sqrt{1+4 b c d^{2}}}{2 d^{2} b}\right)
\end{array}\right\}
$$

Using Routh-Hurwitz criterion, the equation (17) has all roots with negative real parts if and only if the conditions are satisfied as follows

$$
\left.\begin{array}{l}
A>0, \\
A B-C>0, \\
(A B-C) C-A^{2} D>0 \\
D>0
\end{array}\right\}
$$

Since $D=0$ therefore one of Routh-Hurwitz conditions is not satisfied, consequently the system (8) is critical case, the proof is completed.

We explain the difference between the two systems by use of the following table

\begin{tabular}{||c|c|c||}
\hline & system (2) & a new system (system (8)) \\
\hline Equation & $\begin{array}{l}\dot{x}=a(y-x) \\
\dot{y}=c x-x z+u \\
\dot{z}=x y-b z \\
\dot{u}=-d x\end{array}$ & $\begin{array}{c}\dot{x}=a(y-x) \\
\dot{y}=c x-x z+u \\
\dot{z}=x y-b z \\
\dot{u}=z-d u\end{array}$ \\
\hline $\begin{array}{c}\text { Equilibri } \\
\text { um }\end{array}$ & $0(0,0,0,0)$ & $\begin{array}{c}0,0,0,0), p_{1}\left(x_{0}, y_{0}, z_{0}, u_{0}\right) \\
p_{2}\left(x_{1}, y_{1}, z_{1}, u_{1}\right)\end{array}$ \\
\hline $\begin{array}{c}\text { Stability } \\
\text { asymptotically stable when } \\
c<0\end{array}$ \\
$\begin{array}{c}\text { Bifurcatio } \\
\text { n }\end{array}$ & not Hopf bifurcation & $\begin{array}{c}c<0 \\
\text { unstable when } c>0\end{array}$ \\
\hline $\begin{array}{c}\text { Effective } \\
\text { u }\end{array}$ & effective u on the system bifurcation \\
\hline $\begin{array}{c}\text { Dissipativ } \\
\text { e }\end{array}$ & dissipative when $a+b>0$ & not effective u on the system \\
\hline
\end{tabular}

\section{5 - Illustrative Example:}

Example: Investigate the stability of the following new hyperchaotic Pan system: 


$$
\left.\begin{array}{rl}
\dot{x} & =10(y-x) \\
\dot{y} & =20 x-x z+u \\
\dot{z} & =x y-6 z \\
\dot{u} & =z+3 u
\end{array}\right\}
$$

\section{Solution:}

At point origin $a=10, c=20, b=6, d=3$ and linearized system (20) about the equilibrium point $O(0,0,0,0)$ yield the following characteristic equation:

$$
\begin{aligned}
& \lambda^{4}+19 \lambda^{3}+108 \lambda^{2}-3600 \lambda-3600=0 \\
& \text { or } \quad \lambda_{4}=-6 \\
& \lambda^{3}+13 \lambda^{2}-170 \lambda-600=0
\end{aligned}
$$

by using Routh-Hurwitz method on equation (22)we obtain $A=13, C=-600$ So $A>0$ but $C<0$ not satisfied Routh-Hurwitz conditions since $c=20>0$ (by theorem 1,(2)), the system (22) is unstable when $c>0$.

but if $c<0 \quad$ (by theorem 1,(1)), let $\mathbf{c}=\mathbf{- 2}$ then yield the following characteristic equations: $\quad \lambda^{3}+13 \lambda^{2}+50 \lambda+60=0$

where satisfied Routh-Hurwitz conditions, consequently, the system (20) is Asymptotically stable at origin when $c<0$.

To justified this results we found the roots of equations (22), (23) the roots of equation (22) are $\lambda_{1}=-3, \lambda_{2}=-20, \lambda_{3}=10$. while the roots of equation (23) are $\lambda_{1}=-3, \lambda_{2}=-5+\sqrt{5}, \lambda_{3}=-5-\sqrt{5}$.

consequently, the system (20) is asymptotically stable at equilibrium point $O(0,0,0,0)$ if $c<0$ and unstable if $c>0$.

At point $p_{1}$ : The characteristic equation is:

$$
\begin{aligned}
& \lambda^{4}+16 \lambda^{3}+\frac{6547}{108} \lambda^{2}+\frac{4020}{108} \lambda=0 \\
\text { or } \quad & \lambda\left(\lambda^{3}+16 \lambda^{2}+\frac{6547}{108} \lambda+\frac{4020}{108}\right)=0
\end{aligned}
$$

therefore $\lambda_{1}=0$, satisfied remark 4 (Critical Cases) by theorem 3, consequently

the solution of system (20) at the equilibrium point $p_{1}$ is critical case.

\section{6- Conclusion:}


This paper presents a new four dimensional hyperchaotic system, called a new hyperchaotic system. This new hyperchaotic system is different from the system proposed by Liu and Feng in 2009 and also the system proposed by Pan in 2011 , we found the parameter c effective on stability of new system at the point $O(0,0,0,0)$ and has two roots form $\lambda_{1}=-b, \quad \lambda_{2}=-d \quad$ while has root $\lambda_{1}=0$ at the points $p_{1,2}$.

\section{REFERENCES}

[1] Borrelli R.L., Coleman C.S. (1998), Differential Equations, New York., John Wiley and Sons, Inc

[2] Chen A., Lu J., L $\ddot{~}$ J., Yu S.,(2006) ,Generating Hyperchaotic L $\ddot{u}$ Attractor Via State Feedback Control, Journal of Science Direct ,Physica A, No.364,pp.103-110.

[3] Elabbasy E. M. , EL-Dessoky M. M. , (2008), Synchronization and Adaptive Synchronization of Hyperchaotic L $\ddot{u}$ Dynamical System, Academic Journals Inc. No.2,pp.129-141.

[4] Liu M., Feng J., (2009), A new Hyperchaotic System ,Acts Physica Since,Vol.58,No.7pp.4457-4462.

[5] Pan L. , Xu D. ,Zhou W. , (2010), Controlling a Novel Chaotic Attractor Using Linear Feedback, Journal of Information and Computing Science, Vol. 5, No.2,pp. 117-124 .

[6] Pan L. ,Zhou W. , Zhou L. ,Sun K. , (2011), Chaos Synchronization Between Two Different Fractional-Order Hyperchaotic Systems , Journal of Science Direct, Communications Nonlinear Science and Numerical Simulation , Vol. 16 ,pp. 2628-2640 .

[7] Wang G., Zhang X., Zheng Y., Li Y., (2006), A New Modified

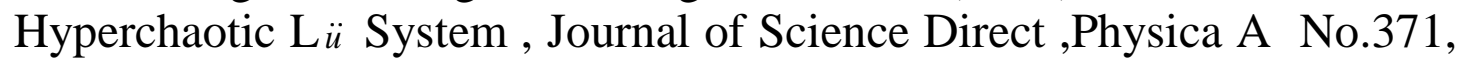
pp.260-272.

[8] Wang G., Zhen Y., Li Y., (2006), Controlling Lorenz System to Hyperchaotic, Proceedings of the 3nd International Conference on impulsive Dynamic System and Applications,pp.1543-1545.

[9] Wang X., Wang M., (2008), A Hyperchaos Generated Form Lorenz System, Journal of Science Direct ,Physica A No.387,pp.37513758.

[10] Xue W. , Mu J., Jia H. , (2010), A Novel One Equilibrium HyperChaotic System and Bifurcation Analysis, International Workshop on Chaos- Fractals Theories and Applications pp. 197-201.

[11] Xu J. , Cai G., Zheng S., (2009), A Novel Hyperchaotic System and Its Control, Journal of Uncertain system, Vol.3,No.2,pp.137-144. 
[12] Yang Q. ,Chen G., (2008), A Chaotic System with One Saddle and Two Stable NOd-Foci, International Journal of Bifurcation and Chaos, Vol.18, No.5, pp.1393--1414.

[13] Yu H., Cai G. , Li Y., (2012), Dynamic Analysis and Control of A New Hyperchaotic Finance System, Nonlinear Dynamic, Springer, No.67, pp. 2171-2182.

[14] Zhuang K.,(2012), Feedback Control Methods for A New Hyperchaotic System, Journal of Information \& Computational Science, Vol.9, No.1, pp.231--237. 